\title{
Exciton localization on basal stacking faults in a-plane epitaxial lateral overgrown GaN grown by hydride vapor phase epitaxy
}

\author{
P. Corfdir, ${ }^{1, a)}$ P. Lefebvre, ${ }^{1,2}$ J. Levrat, ${ }^{1}$ A. Dussaigne, ${ }^{1}$ J.-D. Ganière, ${ }^{1}$ D Martin, ${ }^{1}$ J. Ristić, ${ }^{1}$ \\ T. Zhu, ${ }^{1}$ N. Grandjean, ${ }^{1}$ and B. Deveaud-Plédran \\ ${ }^{1}$ Institut de Photonique et d'Electronique Quantiques, EPFL, 1015 Lausanne, Switzerland \\ ${ }^{2}$ Groupe d'Etude des Semiconducteurs, CNRS-Université de Montpellier II, 34095 Montpellier, France
}

(Received 17 September 2008; accepted 17 December 2008; published online 17 February 2009)

\begin{abstract}
We present a detailed study of the luminescence at $3.42 \mathrm{eV}$ usually observed in $a$-plane epitaxial lateral overgrowth (ELO) GaN grown by hydride vapor phase epitaxy on $r$-plane sapphire. This band is related to radiative recombination of excitons in a commonly encountered extended defect of $a$-plane GaN: $I_{1}$ basal stacking fault. Cathodoluminescence measurements show that these stacking faults are essentially located in the windows and the N-face wings of the ELO-GaN and that they can appear isolated as well as organized into bundles. Time-integrated and time-resolved photoluminescence, supported by a qualitative model, evidence not only the efficient trapping of free excitons (FXs) by basal plane stacking faults but also some localization inside $I_{1}$ stacking faults themselves. Measurements at room temperature show that FXs recombine efficiently with rather long luminescence decay times (360 ps), comparable to those encountered in high-quality GaN epilayers. We discuss the possible role of $I_{1}$ stacking faults in the overall recombination mechanism of excitons. (C) 2009 American Institute of Physics. [DOI: 10.1063/1.3075596]
\end{abstract}

\section{INTRODUCTION}

During the last decade, group-III nitrides have become the materials of choice for solid-state emission of visible-UV light. ${ }^{1}$ However, discontinuities of both the spontaneous and the piezoelectric polarizations at the interfaces of nitride based heterostructures induce a huge electric field along the [0001] direction. The consequences of this electric field on the emission properties are detrimental as it decreases the overlap between the electron and hole wave functions. ${ }^{2}$ This so-called quantum confined Stark effect (QCSE) leads to a spectral redshift and to a dramatic increase in the radiative lifetime, worsening the influence of fast nonradiative processes. The growth of nitride heterostructures along a nonpolar direction (i.e., perpendicular to the $c$-axis) is consequently a way to overcome the QCSE (Ref. 3) and to increase the radiative efficiency. However, the growth of $a$-plane $\mathrm{GaN}$ is complex and still gives rise to large densities of dislocations and stacking faults.

\section{GROWTH PROCEDURE AND EXPERIMENTAL SETUP}

Several groups have recently reported on the fabrication of $a$-plane GaN templates using the epitaxial lateral overgrowth (ELO) technique with improved surface morphology and material quality in the two types of so-called "wing" regions ${ }^{4,5}$ that grow along the $+c$ (Ga-face) and $-c(\mathrm{~N}$-face) directions. We have used a similar approach. First, a $1.7 \mu \mathrm{m}$ thick planar $a$-plane GaN layer was grown on $r$-plane sapphire by hydride vapor phase epitaxy (HVPE). ${ }^{6}$ It was further patterned with $\mathrm{SiO}_{2}$ stripes $(200 \mathrm{~nm}$ thick and $40 \mu \mathrm{m}$ wide) oriented along the [1-100] direction, separated by

${ }^{a)}$ Electronic mail: pierre.corfdir@epfl.ch.
$5 \mu \mathrm{m}$ wide windows. These windows seeded the HVPE growth of a $22 \mu \mathrm{m}$ thick uncoalesced $a$-plane GaN ELO epilayer with ELO-wing widths varying from 20 to $30 \mu \mathrm{m}$.

Cathodoluminescence (CL) microscopic maps were taken at $8 \mathrm{~K}$ with a Cambridge S360 scanning electron microscope (acceleration voltage of $20 \mathrm{kV}$ ) and analyzed through a spectrometer with focal length of $25 \mathrm{~cm}$ and grating with 1200 grooves $/ \mathrm{mm}$. Our time-resolved photoluminescence (TRPL) setup uses the third harmonic of an $\mathrm{Al}_{2} \mathrm{O}_{3}$ :Ti mode-locked laser $(\lambda=280 \mathrm{~nm})$, with pulse width and repetition rate of $2 \mathrm{ps}$ and $80.7 \mathrm{MHz}$, respectively. For these TRPL experiments the samples were cooled in a closed-cycle helium cryostat that can reach a temperature of $8 \mathrm{~K}$. The luminescence of the samples was analyzed by a spectrometer with $27 \mathrm{~cm}$ focal length and a grating with 600 grooves $/ \mathrm{mm}$, followed by a streak camera synchronized with the pulsed laser working in photon counting mode.

\section{CL OF BASAL-PLANE STACKING FAULTS}

\section{A. CL spectrum: Identification of the basal stacking faults}

CL spectra taken at $8 \mathrm{~K}$ show two intense recombination lines in the region near the band edge of GaN [Fig. 1(a)]. The higher energy line lies at $3.471 \mathrm{eV}$ and is related to the recombination of donor-bound excitons $\left(\mathrm{D}^{0} \mathrm{X}\right)$, whereas the $3.417 \mathrm{eV}$ transition arises from the recombination of excitons bound to the so-called $I_{1}$ basal stacking faults (BSFs). ${ }^{7,8}$ Within the hexagonal phase, the atomic planes normally follow the stacking sequence ...AaBbAaBbAaBb... along the [0001] direction (capital and lowercase letters correspond to $\mathrm{Ga}$ and $\mathrm{N}$ atoms, respectively). However, sequences of two elementary stacking faults may appear and they can be seen 

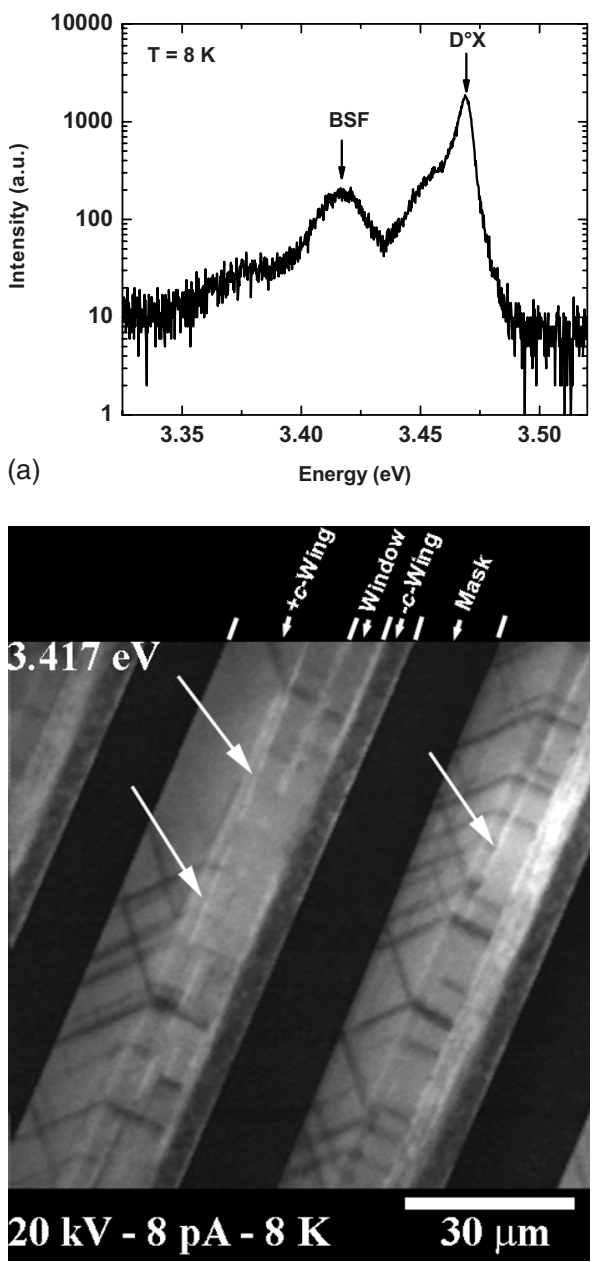

(b)

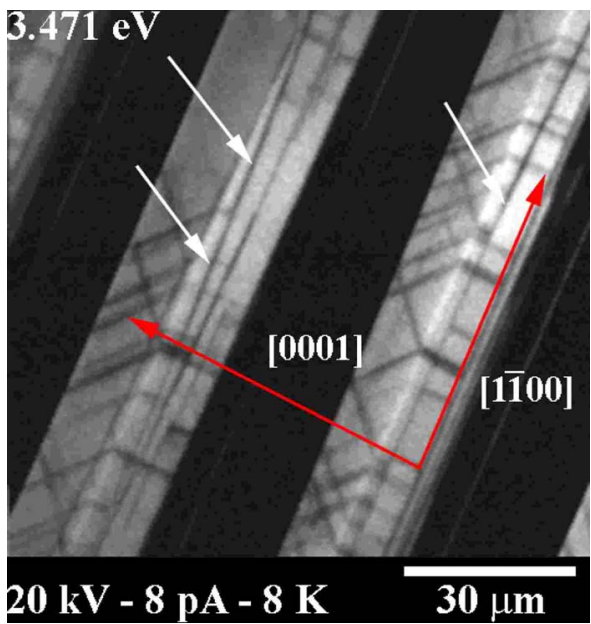

(c)

FIG. 1. (Color online) (a) CL spectrum of $a$-plane ELO-GaN taken at $8 \mathrm{~K}$ with an acceleration voltage of $20 \mathrm{kV}$ and a current of $500 \mathrm{pA}$. [(b) and (c)] $\mathrm{CL}$ maps of ELO $a$-plane GaN taken at the recombination energy of $I_{1}$ BSF-bound excitons $(3.417 \mathrm{eV})$ and neutral donor-bound excitons (3.471 $\mathrm{eV})$. White arrows point out the emergence of dense systems of BSF planes and illustrate the anticorrelation between the emissions at 3.417 and 3.471 eV.

as planar and narrow (a few monolayers thick) inclusions of zinc-blende-like $\mathrm{GaN}$ in the form of $A a B b C c$ sequences that are equivalent to those of cubic GaN along the [111] direction. $^{7,8}$
Type 1 (or $I_{1}$ ) BSFs are produced at the beginning of the growth and then they propagate perpendicularly to the $c$-axis from the substrate toward the surface of the sample. Rarely observed away from the substrate in $c$-plane GaN, BSFs are found in $a$-plane samples with densities as high as $10^{5} \mathrm{~cm}^{-1}$, as already reported by other groups. ${ }^{9}$ Stampfl and Van de Walle $^{10}$ identified four different types of BSFs. The one that we observe here is the $I_{1}$ type, which consists of one violation of the stacking sequence: if the fault starts on a $B b$ layer, the stacking sequence is then ...AaBbAaBbCcBbCcBbAaBb... These BSFs are commonly considered as quantum wells made of zinc-blende-like material surrounded by the wurtzite matrix, giving rise to a luminescence line at lower energy than the excitonic gap of wurtzite GaN. ${ }^{7}$ Density functional pseudopotential calculations ${ }^{10}$ and another model based on elastic deformation potentials ${ }^{11}$ both suggest that the valence- and conduction-band offsets between wurtzite and zinc blende should generate a shallow and thin type-II quantum well $\left[\Delta E_{C}=122 \mathrm{meV}, \Delta E_{V}=-62 \mathrm{meV}\right.$, and $L=0.77 \mathrm{~nm}$ (Ref. $7)$, where electrons are only confined within the $I_{1}$ BSF. Then the hole would face a potential barrier in this zincblende-like layer and it would therefore be maintained in the surrounding wurtzite area but in the vicinity of the quantum well because of the Coulomb attraction that binds it to the electron. Such a type-II exciton can be modeled efficiently by the so-called "effective potential" method" ${ }^{12}$ which is a mathematical approximation based on the variational theorem, where everything turns as if the hole was confined in an effective (not real) triangular potential. Then the electronhole pair can recombine radiatively solely through thepresumably weak - overlap of their probability density distributions. Quite importantly for the following discussion of experimental results, a spectrally defined optical recombination exists only because of the Coulomb binding. Thermal excitation is, in principle, able to ionize this type-II exciton, pushing it into the continuum of its scattering (unbound) states. One may be tempted to describe this situation as the escape of the hole out of its triangular well, but this is a simplistic view, since the triangular well is really a mathematical approximation and not a real potential well.

\section{B. Monochromatic CL mapping: Localization of the BSFs}

In terms of spatial distribution of the BSFs, previous studies by transmission electron microscopy ${ }^{13}$ reported that their density is higher by two orders of magnitude in the $-c$ ELO wing and in the window region than in the $+c$ wing. Our top view monochromatic CL map taken for emission at $3.417 \mathrm{eV}$, shown in Fig. 1(b), confirms that intense emission related to BSFs comes from the $-c$ ELO wings, where it presents a rather spotty distribution and, above all, ${ }^{13}$ from the window region, where it has the aspect of dashes oriented along the $c$-plane.

However, the $3.417 \mathrm{eV}$ emission can also be excited in the $+c$ wing: the most salient contribution (also observed in Ref. 14) is shown by arrows in Figs. 1(b) and 1(c), in the form of narrow regions, elongated over several micrometers, perpendicular to the [0001] direction. In these regions, the 


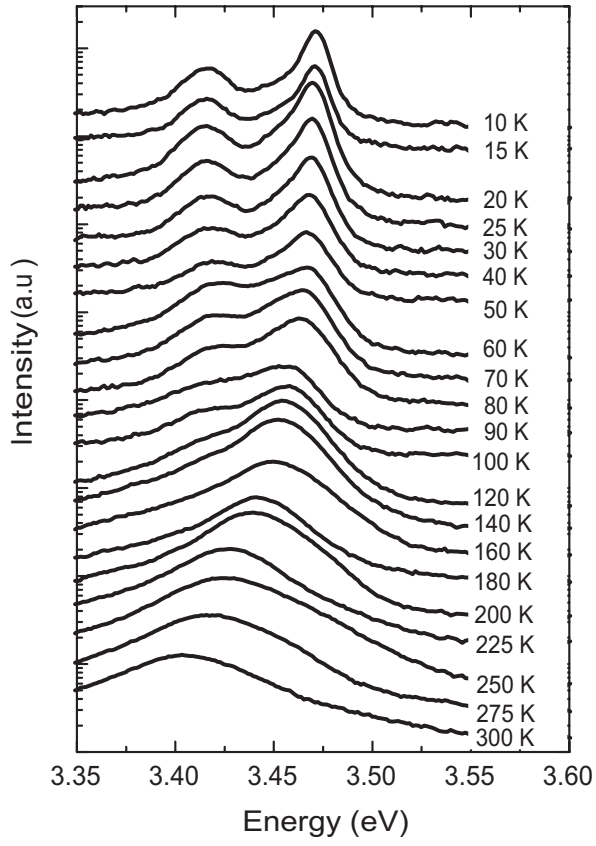

FIG. 2. Time-integrated photoluminescence spectra of $a$-plane ELO-GaN taken at different temperatures. The spectra have been shifted vertically for clarity.

intense emission related to BSFs is clearly anticorrelated with the emission at $3.471 \mathrm{eV}$, related to $\mathrm{D}^{0} \mathrm{X}$ recombination. Nevertheless, Fig. 1(b) also shows that a weak signal is detected at $3.417 \mathrm{eV}$ over the entire $+c$ wing: the emission related to the BSFs is excited even when the electronic excitation beam is focused $10 \mu \mathrm{m}$ away from the elongated regions of intense BSF-related emission. One may be tempted to attribute this emission to carriers that would be scattered from the excitation spot to the regions where intense BSF recombination is observed. However, the estimation of the excitation volume for our $20 \mathrm{kV}$ electron beam with Monte Carlo simulations, ${ }^{15}$ combined with the experimental determination of the diffusion length of excited carriers in $\mathrm{GaN},{ }^{16}$ shows that carriers only diffuse $\sim 1.5 \mu \mathrm{m}$ away from the excitation spot.

To summarize these observations, we deduce that the $+c$ wing in our sample contains a nearly homogeneous distribution of BSFs. But, in addition to this distribution, the emergence of bundles of $I_{1}$ BSFs appears in the form of bright elongated regions, where the emission of $\mathrm{D}^{0} \mathrm{X}$ is inhibited. Similar bundles, of apparently shorter extension along the $c$-plane, are visible in very high densities in the window region and, to a lesser extent, in the $-c$ wing. In addition to $I_{1}$ BSFs, our CL maps also reveal the emergence of another type of planar defects that appear as dark lines even in polychromatic CL. The properties of these nonradiative traps that we relate to prismatic stacking faults will be discussed elsewhere.

\section{LOCALIZATION EFFECTS}

Time-integrated PL spectra have been recorded between 10 and $300 \mathrm{~K}$ (Fig. 2). At $10 \mathrm{~K}$, we can distinguish the two intense lines at 3.471 and $3.417 \mathrm{eV}$ related to donor-bound and BSF-bound excitons, respectively. On the high-energy

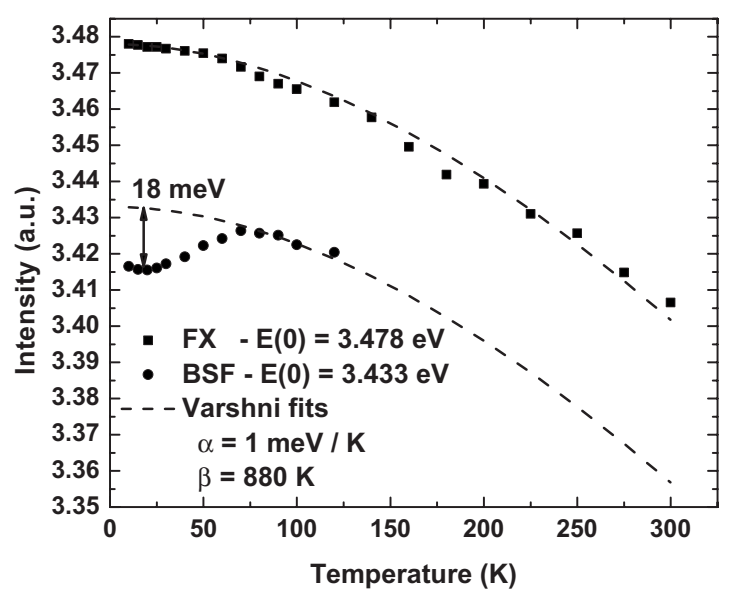

FIG. 3. Energy position vs temperature of the free- and BSF-bound exciton lines (square and circle symbols, respectively). Dashed curves correspond to the result of Varshni's semiempirical model.

side of the $\mathrm{D}^{0} \mathrm{X}$ line lies the contribution from the recombination of the A free exciton (FX) at $3.478 \mathrm{eV}$, which has to be taken into account in a careful deconvolution procedure, since it will progressively dominate the PL spectrum when the temperature is increased. The emission line related to BSFs presents a full width at half maximum (FWHM) of 30 $\mathrm{meV}$, which is large when compared to the $\mathrm{D}^{0} \mathrm{X}$ line (FWHM of $7 \mathrm{meV}$ ). As already reported for $c$-plane HVPE GaN, ${ }^{17}$ our unintentionally $n$-doped samples also contain a residual doping by acceptors, which may give rise to a weaker emission at $3.465 \mathrm{eV}$ related to acceptor bound exciton $\mathrm{A}\left(\mathrm{A}^{0} \mathrm{X}\right)$.

Figure 3 displays the temperature dependence of the FX and BSF energies obtained by deconvolution of the spectra in Fig. 2. Experimental points are compared with the result of Varshni's empirical formula $E(T)=E(0)-\alpha T^{2} /(T+\beta)$. For the FX, we obtained the best fit for $E(0)=3.478 \mathrm{eV}, \alpha$ $=0.80 \mathrm{meV} / \mathrm{K}$, and $\beta=800 \mathrm{~K}$. These values are in the range of those reported for $c$ - and $a$-plane GaN. ${ }^{18}$ At low temperature, A excitons are essentially localized on donors but they escape when the temperature is increased above $\sim 50 \mathrm{~K}$. In agreement with previous observations in $c$-plane ${ }^{19}$ and $a$-plane $\mathrm{GaN},{ }^{20}$ the BSF energy position follows an "Sshaped" temperature dependence consisting in a small redshift from 8 to $25 \mathrm{~K}$ followed by a blueshift that eventually merges with some Varshni curve for temperatures above 75 K.

Given the fact that the wave functions of excitons bound to BSFs are quite extended in space (see discussion below), then BSF-bound excitons should be sensitive to potential fluctuations that could be induced, for instance, by the random distribution of extrinsic donors located in the vicinity of the BSF or by strain fluctuations. ${ }^{21}$ There are consequently some localization effects for BSF-bound excitons inside the stacking faults themselves, explaining qualitatively the redshift with temperature from 8 to $25 \mathrm{~K}$ due to the ionization of excitons bound to the shallower potential fluctuation in favor of the deeper ones. This explanation is also consistent with the 3-4 meV blueshift and the vanishing of the low-energy tail of the BSF-related emission observed by Paskov et al. ${ }^{22}$ when increasing the excitation density. 
The blueshift part of the S-shaped temperature dependence is commonly observed in epitaxial type-I and type-II quantum wells ${ }^{23,24}$ due to fluctuations of the well width by one or two monolayers. However, it is unlikely that quantum wells formed by stacking faults present such fluctuations because the distance between two changes in the stacking sequence is precisely determined by the type of the BSF: for instance, the width of $I_{1}$ stacking faults is exactly equal to $1.5 c_{0}$, where $c_{0}$ is the unstrained lattice parameter of wurtzite GaN along the (0001) direction. Paskov et al. ${ }^{22}$ attributed similar temperature dependence of the BSF-related line to the delocalization of the holes from the potential where they are maintained in the type-II quantum well by Coulomb attraction to the electron. In other words, the blueshift would be due to a transition from an exciton bound to the BSF to a free-to-bound recombination involving a bound electron and a free hole. According to this reasoning, the measured hole delocalization energy would be nothing but the binding energy of the type-II exciton. We measure $18 \mathrm{meV}$ for this localization energy, which is the difference between the lowtemperature extrapolated PL energy of $\sim 3.433 \mathrm{eV}$ and the measured minimum PL energy of $3.415 \mathrm{meV}$. Paskov et al. ${ }^{22}$ reported $\sim 20 \mathrm{meV}$, instead. One explanation for this difference could be in a difference of internal electric fields, which Paskov et l. $^{22}$ did not consider when discussing the hole escape from the Coulomb binding. Such fields, which could arise from different doping concentrations ${ }^{25}$ or different distances between successive BSFs, ${ }^{26}$ certainly exist in and around such BSFs, whatever their type-I or type-II alignment, as commented by Sun et al. ${ }^{27}$ Now, a binding energy of $18 \mathrm{meV}$ seems quite small compared to the bulk value (26 $\mathrm{meV}$ ) for a type-II exciton in such shallow and narrow quantum wells, especially if one considers the presence of electric fields that, for type-II structures, tend to minimize the electron-hole separation (see Fig. 10 of Ref. 27). In fact, somewhat paradoxically, we may even have here an increase in the exciton binding energy compared to the bulk value since we have a nearly bidimensional situation.

To be completed-if not conclusive-we must also mention one suggestion by Paskov et $a l^{22}$ that the BSF-related emission may arise from bundles of BSFs. As shown above, the distribution of BSFs is highly nonuniform: single BSFs are probably present in the $+c$ wings, whereas bundles of BSFs, with various densities and extensions, are observed in all regions. These bundles of BSFs could behave like coupled quantum wells, provided that the $I_{1}$ stacking faults are packed closely enough. Considering the thinness $(0.77$ $\mathrm{nm})$ and the depth of the wells $\left(\Delta E_{C}=122 \mathrm{meV}\right)$ the electron wave functions can easily leak out into the wurtzite barriers, making the coupling possible. The single energy levels of each quantum well would thus be split into many levels organized in minibands due to the overlap of the wave functions. Using the electron wave function calculated by Rebane et al. ${ }^{11}$ we find a penetration length of the electron wave function inside the hexagonal phase of $\sim 3 \mathrm{~nm}$, a value compatible with quantum coupling between two BSFs as their average separation distance in both the window and the $-c$ wings can be estimated as $10 \mathrm{~nm} .{ }^{14}$

The temporal evolution of PL spectra brings additional

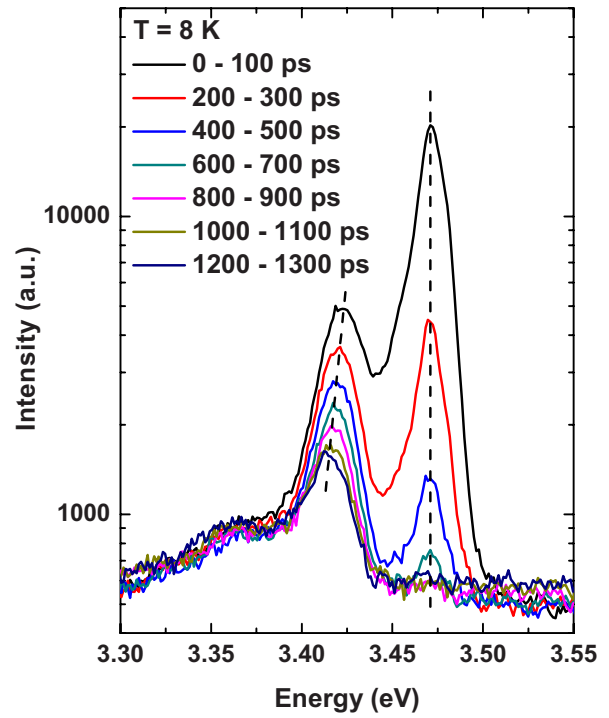

FIG. 4. (Color online) Evolution with time of the PL spectrum of our ELO $a$-plane GaN taken at $8 \mathrm{~K}$. Dashed curves are guides for the eyes.

insight into localization effects inside the BSFs (Fig. 4). During the first nanosecond of decay, we observe a narrowing of the BSF-related emission from 26 to $18.5 \mathrm{meV}$, and the highenergy tail becomes steeper, resulting in a spectral redshift of the BSF emission from $3.424 \mathrm{eV}$ at the beginning of the decay to $3.415 \mathrm{eV}$ after $1 \mathrm{~ns}$. Decreasing the excitation density by two orders of magnitude does not result in any change in this time dependence of the BSF-related line (not shown here), indicating that the observed behavior does not result from some "descreening" of internal electric fields ${ }^{28}$ or from band filling. ${ }^{29}$ In contrast, this redshift is consistent with the existence of different degrees of localization that can originate either from a miniband formed by coupled BSFs or from extrinsic localization centers such as donors in the vicinity of the BSFs. During the first hundred picoseconds after the excitation pulse, BSF excitons are distributed over a large density of state, resulting in an emission line centered at $3.424 \mathrm{eV}$ with a FWHM of $26 \mathrm{meV}$. The higherenergy BSF-bound excitons are less localized and recombine faster than those localized deeper resulting, during the first nanosecond of decay, in the vanishing of the high-energy tail of the emission. The redshift and the narrowing observed would then result from the difference in lifetime between shallow and deep potential fluctuations. The smaller lifetime observed at higher energy certainly results from both an intrinsically shorter radiative lifetime (weaker localization) and from transfer and capture mechanisms toward deeper potential fluctuations. To support this interpretation, we remark that the BSF-related emission at $10 \mathrm{~K}$ after $1 \mathrm{~ns}$ of decay lies at the same (minimum) energy as the time-integrated BSFrelated emission measured at $25 \mathrm{~K}$ (Fig. 3). The latter indeed results from the thermally enhanced transfer of excitons bound to shallow potential fluctuations toward deeper ones.

\section{CAPTURE DYNAMICS OF FX IN A-PLANE GAN}

Time-resolved PL experiments have been performed in order to get further insight into the transfer dynamics of FXs 


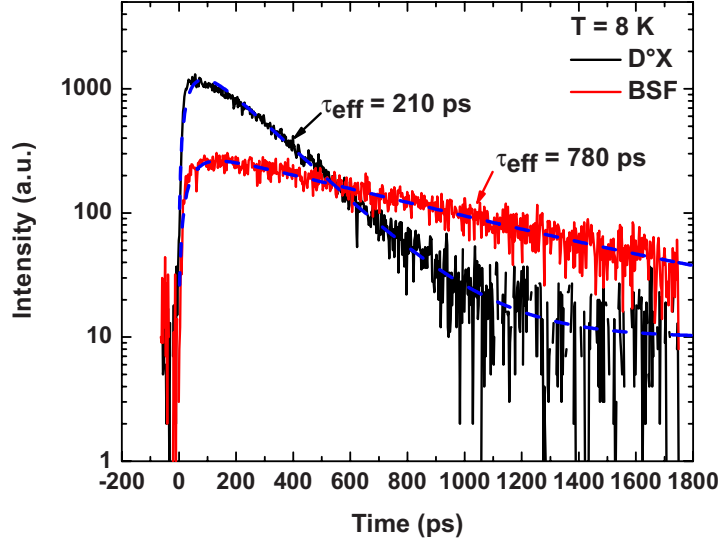

FIG. 5. (Color online) Luminescence decays of neutral donor-bound (black) and BSF-bound (red) exciton recombinations at $8 \mathrm{~K}$ for our $a$-plane ELO$\mathrm{GaN}$ sample. Dashed curves are the result of our rate-equation model.

toward neutral donors and BSFs. We have recorded, at $T$ $=8 \mathrm{~K}, \mathrm{PL}$ decay times of $210 \mathrm{ps}$ for the $\mathrm{D}^{0} \mathrm{X}$ recombination line and 780 ps for BSF-bound excitons (Fig. 5). Furthermore the rise time of the BSF-related luminescence is longer than that of the $\mathrm{D}^{0} \mathrm{X}$ recombination and the luminescence decays of these two transitions get faster for delays larger than $120 \mathrm{ps}$ after the excitation pulse. This indicates that the capture of FXs by BSFs and donors is more efficient than radiative recombinations of these excitons once they are bound, resulting in slower effective decays of $\mathrm{D}^{0} \mathrm{X}$ and $\mathrm{BSF}$ at short delays after the pulses.

Considering that the population of FXs generated just after the excitation pulse can be trapped by the donors and by the BSFs, we write

$$
\begin{aligned}
\frac{d n_{\mathrm{FX}}}{d t}= & -\frac{n_{\mathrm{FX}}}{\tau_{\mathrm{r}, \mathrm{FX}}}-\frac{n_{\mathrm{FX}}}{\tau_{\mathrm{FX} \rightarrow \mathrm{D}^{0} \mathrm{X}}}-\frac{n_{\mathrm{FX}}}{\tau_{\mathrm{FX} \rightarrow \mathrm{BSF}}}+\frac{n_{\mathrm{BSF}}}{\tau_{\mathrm{BSF} \rightarrow \mathrm{FX}}} \\
& +\frac{n_{\mathrm{D}^{0} \mathrm{X}}}{\tau_{\mathrm{D}^{0} \mathrm{X} \rightarrow \mathrm{FX}}}-\frac{n_{\mathrm{FX}}}{\tau_{\mathrm{nr}, \mathrm{FX}}},
\end{aligned}
$$

where $n_{\mathrm{FX}}$ is the population of FXs and $\tau_{\mathrm{r}, \mathrm{FX}}$ and $\tau_{\mathrm{nr}, \mathrm{FX}}$ are their radiative and nonradiative lifetimes. FXs are trapped by donors and BSFs with characteristic times denoted $\tau_{\mathrm{FX} \rightarrow \mathrm{D}^{0} \mathrm{X}}$ and $\tau_{\mathrm{FX} \rightarrow \mathrm{BSF}}$, respectively. Detrapping processes from donors and BSFs are taken into account by the following characteristic times: $\tau_{\mathrm{D}^{0} \mathrm{X} \rightarrow \mathrm{FX}}, \tau_{\mathrm{BSF} \rightarrow \mathrm{FX}}, \tau_{\mathrm{D}^{0} \mathrm{X} \rightarrow \mathrm{BSF}}$, and $\tau_{\mathrm{BSF} \rightarrow \mathrm{D}^{0} \mathrm{X}}$. EXcitons bound to donors or to BSFs can then decay either

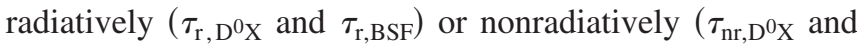
$\left.\tau_{\mathrm{nr}, \mathrm{BSF}}\right)$,

$$
\begin{aligned}
\frac{d n_{\mathrm{D}^{0} \mathrm{X}}}{d t}= & -\frac{n_{\mathrm{D}^{0} \mathrm{X}}}{\tau_{\mathrm{r}, \mathrm{D}^{0} \mathrm{X}}}+\frac{n_{\mathrm{FX}}}{\tau_{\mathrm{FX} \rightarrow \mathrm{D}^{0} \mathrm{X}}}-\frac{n_{\mathrm{D}^{0} \mathrm{X}}}{\tau_{\mathrm{nr}, \mathrm{D}^{0} \mathrm{X}}}-\frac{n_{\mathrm{D}^{0} \mathrm{X}}}{\tau_{\mathrm{D}^{0} \mathrm{X} \rightarrow \mathrm{FX}}} \\
& -\frac{n_{\mathrm{D}^{0} \mathrm{X}}}{\tau_{\mathrm{D}^{0} \mathrm{X} \rightarrow \mathrm{BSF}}}+\frac{n_{\mathrm{BSF}}}{\tau_{\mathrm{BSF} \rightarrow \mathrm{D}^{0} \mathrm{X}}},
\end{aligned}
$$

$$
\begin{aligned}
\frac{d n_{\mathrm{BSF}}}{d t}= & -\frac{n_{\mathrm{BSF}}}{\tau_{\mathrm{r}, \mathrm{BSF}}}+\frac{n_{\mathrm{FX}}}{\tau_{\mathrm{FX} \rightarrow \mathrm{BSF}}}-\frac{n_{\mathrm{BSF}}}{\tau_{\mathrm{nr}, \mathrm{BSF}}}-\frac{n_{\mathrm{BSF}}}{\tau_{\mathrm{BSF} \rightarrow \mathrm{FX}}} \\
& +\frac{n_{\mathrm{D}^{0} \mathrm{X}}}{\tau_{\mathrm{D}^{0} \mathrm{X} \rightarrow \mathrm{BSF}}}-\frac{n_{\mathrm{BSF}}}{\tau_{\mathrm{BSF} \rightarrow \mathrm{D}^{0} \mathrm{X}}} .
\end{aligned}
$$

As exciton polaritons are stationary states of infinite dielectric medium, they should not exhibit any temporal evolution: the radiative decay of FXs should consequently be much slower than the capture processes in which we are interested. Moreover, nonradiative and delocalization processes are neglected as they should not be dominant at $8 \mathrm{~K}$, which simplifies the model. To further reduce the number of fitting parameters, we have also neglected, in first approximation, the direct capture of exciton from neutral donors into BSFs even though we have proposed earlier that the proximity of donors can be a mechanism of localization. Solving analytically the simplified differential equations [Eqs. (1)-(3)], and using them to fit experimental results, yields $\tau_{\mathrm{r}, \mathrm{D}^{0} \mathrm{X}}$ and $\tau_{\mathrm{r}, \mathrm{BSF}}$ equal to 215 and $770 \mathrm{ps}$, respectively. As it can be expected, these values are close to the effective decay times of the two transitions measured at long delays since we have neglected nonradiative recombinations. By fitting the luminescence rise times of the $\mathrm{D}^{0} \mathrm{X}$ and $\mathrm{BSF}$ lines, we also found capture times of FXs by donors and BSFs of 70 and 120 ps, respectively. However, we want to emphasize that in contrast with the concentration of donors that should be homogeneous all over the sample, the BSFs are characterized by the superposition of some homogeneous distribution and of bundles, essentially located in the $-c$ wings and in the window region of the present ELO-GaN layer. As our excitation laser spot has a $40 \mu \mathrm{m}$ diameter, the $120 \mathrm{ps}$ capture time of FXs by BSFs necessarily represents an average of the different capture dynamics that take place in the $-c$ and $+c$ wings and in the window region, where FXs are affected differently by the presence of stacking faults in various densities. For instance, when FXs are generated in the vicinity of a high density of BSFs, their capture by these extended defects is much more probable than that by donors. This result is in total agreement with CL maps in Fig. 1 where we see the inhibition of the emission by donors when the electron beam is focused to the emergence of bundles of BSFs. Nevertheless, we remark that the latter behavior could as well be assigned to the direct transfer of excitons from donors into BSFs that we have neglected in our simplified model. Further investigation by, e.g., time-resolved CL, may provide evidence on whether or not the mechanism of direct capture from donors exists.

Rising the temperature from 8 to $300 \mathrm{~K}$ opens nonradiative channels and delocalizes excitons from the donors. Even in high-quality ELO $c$-plane GaN epilayers, dramatic decreases in both intensity and effective lifetime of the excitonic luminescence are observed due to the dominance of nonradiative recombinations when the temperature is increased. For instance, an effective lifetime of 86 ps was reported for FXs at $300 \mathrm{~K}$ in $c$-plane ELO-GaN (Ref. 30) and we also obtain similar lifetimes in the $60 \mathrm{ps}$ range on comparable samples. Yet, it is not the case in our $a$-plane $\mathrm{GaN}$ sample where we observe for excitonic luminescence an effective lifetime of $360 \mathrm{ps}$ at room temperature (Fig. 6). 


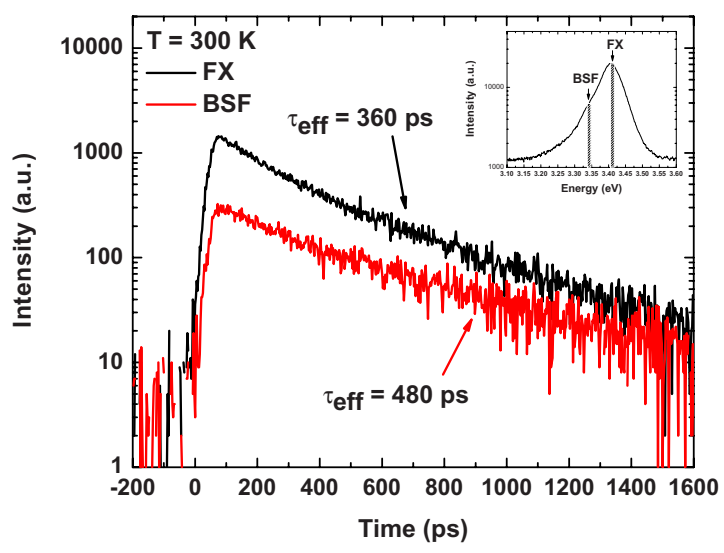

FIG. 6. (Color online) Luminescence decays of free- (black) and BSFbound (red) excitonic recombination taken at $300 \mathrm{~K}$ for $a$-plane ELO-GaN sample. Inset: time-integrated PL spectrum at $300 \mathrm{~K}$. Dashed energy intervals show at which energy the PL decays were recorded.

Chichibu et al. $^{31}$ also reported a long-lived excitonic luminescence of $150 \mathrm{ps}$ in nonpolar $\mathrm{GaN}$ that they attributed to the high crystalline quality of their freestanding $m$-plane GaN, where the BSF density is lower than $10^{3} \mathrm{~cm}^{-1}$. This can definitely not be the case in our ELO-GaN where excitons are nevertheless apparently protected from nonradiative processes at temperature as high as $300 \mathrm{~K}$. The shallow donors and acceptors cannot protect excitons from nonradiative recombination at this temperature since both $\mathrm{D}^{0} \mathrm{X}$ and $\mathrm{A}^{0} \mathrm{X}$ are fully ionized (localization energies of 7 and $12 \mathrm{meV}$, respectively). This is however not exactly the case for the BSFs, for which the localization energy of $61 \mathrm{meV}$ is much larger than $k T$ even at $300 \mathrm{~K}$. The broad spectrum taken at $300 \mathrm{~K}$ (inset of Fig. 6) shows that carrier thermalization is certainly achieved between BSF bound and FXs. The lowenergy PL probably results, by the way, from the superposition of the BSF-related signal and from the phonon-assisted satellite transitions related to FXs. But, whatever their detailed origin, the decay dynamics of the high- and lowenergy regions are similar, especially at long delays, and they are rather slow. We conclude that a constant exchange of carriers between the energy levels free- and BSF-bound excitons takes place via the nearly exact compensation of carrier trapping and release processes between the two levels. As a result of this permanent exchange, a large portion of delocalized carriers remain close to the stacking faults and the long-lived luminescence that we collect does not really originate from completely FXs but rather from a population of excitons that remains in the vicinity of the BSFs. Long excitonic lifetime at room temperature in nonpolar $\mathrm{GaN}$ is consequently due to the fact that BSFs prevent excitons from coming across nonradiative recombination centers.

\section{CONCLUSIONS}

We have investigated the $3.417 \mathrm{eV}$ emission line characteristic of the $I_{1}$ basal stacking faults in an $a$-plane ELO-GaN sample grown by HVPE on $r$-plane sapphire. These extended radiative defects, consisting in a few monolayers of zincblende-like GaN embedded in wurtzite GaN, are nearly homogeneously distributed in the $+c$ wings. However, in these regions and, to a larger extent in the $-c$ wings and so-called "window" regions, the BSFs tend to organize into bundles. Time and temperature dependent PL measurements evidence localization processes inside the BSFs themselves. Contrary to what usually happens in quantum wells, this localization cannot be due to well width fluctuation. We therefore suggest that either quantum coupling between BSFs or a randomly distributed population of extrinsic donors in the vicinity of BSFs could be at the origin of such an exciton localization. Measurements at room temperature show that free- and BSFbound excitons constitute a thermalized system. Large luminescence decay times of $360 \mathrm{ps}$ for the near band-edge emission of our $a$-plane $\mathrm{GaN}$ is consequently assigned to the presence of BSFs that protect FXs from nonradiative recombination.

\section{ACKNOWLEGMENTS}

The authors wish to thank P. Stadelmann and G. Salviati for fruitful discussions and R. Rochat and N. Leiser for technical assistance. IPEQ-EPFL acknowledges financial support from the Swiss National Science Foundation and from the Swiss NCCR research program Quantum Photonics.

${ }^{1}$ S. Nakamura, T. Mukai, and A. Senoh, Appl. Phys. Lett. 64, 1687 (1994). ${ }^{2}$ M. Leroux, N. Grandjean, M. Laügt, J. Massies, B. Gil, P. Lefebvre, and P. Bigenwald, Phys. Rev. B 58, R13371 (1998).

${ }^{3}$ N. Akopian, G. Bahir, D. Gershoni, M. D. Craven, J. S. Speck, and S. P. DenBaars, Appl. Phys. Lett. 86, 202104 (2005).

${ }^{4}$ X. Ni, Ü. Özgür, Y. Fu, N. Biyikli, J. Xie, A. A. Baski, H. Morkoç, and Z. Liliental-Weber, Appl. Phys. Lett. 89, 262105 (2006).

${ }^{5}$ B. Imer, F. Wu, S. P. DenBaars, and J. S. Speck, Appl. Phys. Lett. 88, 061908 (2006).

${ }^{6}$ T. Zhu, D. Martin, R. Butté, J. Napierala, and N. Grandjean, J. Cryst. Growth 300, 186 (2007).

${ }^{7}$ G. Salviati, M. Albrecht, C. Zanotti-Fregonara, N. Armani, M. Mayer, Y. Shreter, M. Guzzi, Y. V. Melnik, K. Vassilevski, V. A. Dmitiev, and H. P. Strunk, Phys. Status Solidi A 171, 325 (1999).

${ }^{8}$ R. Liu, A. Bell, F. A. Ponce, C. Q. Chen, J. W. Yang, and M. A. Khan, Appl. Phys. Lett. 86, 021908 (2005).

${ }^{9}$ X. Ni, U. Özgür, Y. Fu, N. Biyikli, J. Xie, A. A. Baski, H. Morkoç, and Z. Liliental-Weber, Appl. Phys. Lett. 89, 262105 (2006).

${ }^{10}$ C. Stampfl and C. G. Van de Walle, Phys. Rev. B 57, R15052 (1998).

${ }^{11}$ Y. T. Rebane, Y. G. Shreter, and M. Albrecht, Phys. Status Solidi A 164, 141 (1997).

${ }^{12}$ A. Bellabchara, P. Lefebvre, P. Christol, and H. Mathieu, Phys. Rev. B 50, 11840 (1994).

${ }^{13}$ T. Gühne, Z. Bougria, P. Venneguès, M. Leroux, and M. Albrecht, J. Appl. Phys. 101, 113101 (2007).

${ }^{14}$ C. Netzel, T. Wernicke, U. Zeimer, F. Brunner, M. Weyers, and M. Kneissl, J. Cryst. Growth 310, 8 (2008).

${ }^{15}$ Simulation done with CASINO, Version 2.42 .

${ }^{16}$ S. Sonderegger, E. Feltin, M. Merano, A. Crottini, J. F. Carlin, R. Sachot, B. Deveaud, N. Grandjean, and J. D. Ganière, Appl. Phys. Lett. 89, 232109 (2006).

${ }^{17}$ D. Martin, J. Napierala, M. Ilegems, R. Butté, and N. Grandjean, Appl. Phys. Lett. 88, 241914 (2006).

${ }^{18}$ I. Vurgaftman and J. R. Meyer, J. Appl. Phys. 94, 3675 (2003).

${ }^{19}$ S. Fischer, G. Steude, D. M. Hofmann, F. Kurth, F. Anders, M. Topf, B. K. Meyer, F. Bertram, M. Schmidt, J. Christen, L. Eckey, J. Holst, A. Hoffmann, B. Mensching, and B. Rauschenbach, J. Cryst. Growth 189-190, 556 (1998).

${ }^{20}$ P. P. Paskov, R. Schifano, T. Paskova, T. Malinauskas, J. P. Bergman, B. Monemar, S. Figge, and D. Hommel, Physica B 376-377, 473 (2006).

${ }^{21}$ B. Bastek, F. Bertram, J. Christen, T. Wernicke, M. Weyers, and M. Kneissl, Appl. Phys. Lett. 92, 212111 (2008).

${ }^{22}$ P. P. Paskov, R. Schifano, B. Monemar, T. Paskova, S. Figge, and D. Hommel, J. Appl. Phys. 98, 093519 (2005).

${ }^{23}$ Y.-H. Cho, G. H. Gainer, A. J. Fischer, J. J. Song, S. Keller, U. K. Mishra, 
and S. P. DenBaars, Appl. Phys. Lett. 73, 1370 (1998).

${ }^{24}$ M. Dinu, J. E. Cunningham, F. Quochi, and J. Shah, J. Appl. Phys. 94, 1506 (2003).

${ }^{25}$ A. Thamm, O. Brandt, J. Ringling, A. Trampert, K. H. Ploog, O. Mayrock, H.-J. Wünsche, and F. Henneberger, Phys. Rev. B 61, 16025 (2000).

${ }^{26}$ M. Leroux, N. Grandjean, J. Massies, B. Gil, P. Lefebvre, and P. Bigenwald, Phys. Rev. B 60, 1496 (1999).

${ }^{27}$ Y. J. Sun, O. Brandt, U. Jahn, T. Y. Liu, A. Trampert, S. Cronenberg, S.
Dhar, and K. H. Ploog, J. Appl. Phys. 92, 5714 (2002).

${ }^{28}$ P. Lefebvre, S. Kalliakos, T. Bretagnon, P. Valvin, T. Talierco, B. Gil, N. Grandjean, and J. Massies, Phys. Rev. B 69, 035307 (2004).

${ }^{29}$ K. Kash and J. Shah, Appl. Phys. Lett. 45, 401 (1984).

${ }^{30}$ T. Izumi, Y. Narukawa, K. Okamoto, Y. Kawakami, Sg. Fujita, and S. Nakamura, J. Lumin. 87-89, 1196 (2000).

${ }^{31}$ S. F. Chichibu, H. Yamaguchi, L. Zhao, M. Kubota, K. Okamoto, and H. Ohta, Appl. Phys. Lett. 92, 091912 (2008). 